\title{
Estimation of Combining Ability and Genetic Components for Yield Contributing Traits in Spring Barley under Normal and Salinity Conditions
}

\author{
E. Mansour ${ }^{\#}$ and E. S. A. Moustafa* \\ Crop Science Department, Faculty of Agriculture, Zagazig \\ University, Zagazig 44519 and ${ }^{*}$ Genetic Resources \\ Department, Desert Research Center, Cairo 11753, Egypt.
}

$\mathbf{H}^{\prime}$ ALF diallel analysis among diverse eight six-rowed hulled spring barley genotypes was performed to provide information on general and specific combining ability, gene action and heritability for different agronomic traits under normal and salinity conditions. The genotypes used were four commercially cultivars (Giza 123, Giza 126, Giza 132 and Giza 2000) and the other four were introduced from ICARDA (Australian, CHK 38, CHK 2 and CHK 53). The parents were grown and crossed during 2014-2015 in the Experimental Farm of Faculty of Agriculture (Ghazala), Zagazig University, Egypt. In the second season 36 genotypes i.e. 28 hybrid combination and eight parents were sown at two distinct locations. The first was in Ghazala as normal condition and the other was under salinity condition in the Experimental Farm of Desert Research Center, Ras-Sudr Research Station, South Sinai, Egypt, with salinity in irrigation water and soil containing $4500 \mathrm{ppm}$ and $5535 \mathrm{ppm}$, respectively. All field experiments lay out in a randomized complete block design with three replications. The measured traits were; plant height $(\mathrm{cm})$, spike length $(\mathrm{cm})$, number of spikes per plant, number of grains per spike, grain weight per spike $(\mathrm{g}), 100$ grain weight $(\mathrm{g})$ and grain yield per plant $(\mathrm{g})$. Analysis of variance indicated that there were highly significant differences between parental genotypes and theirs F1 crosses for all studied traits. General (GCA) and specific (SCA) combining ability effects were highly significant under both conditions for all traits. The parental genotypes; P3 (Australian) and P6 (CHK 53) showed good performance as well as good GCA effects under salinity condition, while P4 (CHK 38) and P8 (Giza 2000) under normal one and P2 (Giza 126), P5 (CHK 2) and P7 (Giza 132) under both conditions. The cross combinations; $\mathrm{P} 3 \times \mathrm{P} 5, \mathrm{P} 3 \times \mathrm{P} 8$ and $\mathrm{P} 5 \times \mathrm{P} 8$ exhibited good performance and significant positive SCA effects for grain yield and its attributes under salinity, while $\mathrm{P} 2 \times \mathrm{P} 7$, $\mathrm{P} 3 \times \mathrm{P} 4, \mathrm{P} 4 \times \mathrm{P} 7, \mathrm{P} 5 \times \mathrm{P} 7 \mathrm{P} 6 \times \mathrm{P} 7$ and $\mathrm{P} 7 \times \mathrm{P} 8$ under both conditions. The variance due to SCA was higher than that of GCA for all the traits except plant height and number of spikes per plant under salinity condition and 100 grain weight under normal condition. The components of genetic variance suggested more contribution of the dominance effects in the inheritance of the studied traits compared to 


\begin{abstract}
additive ones. Narrow sense heritability values changed from environment to another, it ranged from low to moderate $(4.62 \%$ to 31.96). Wr-Vr graphs showed high degree of genetic diversity for parents with different degrees of dominance and different distribution of dominant and recessive alleles in the parental material for all traits under both conditions.
\end{abstract}

Keywords: Spring barley, Salinity stress, Diallel analyses, Gene action, Wr-Vr Graph.

Barley (Hordeum vulgare L.) is one of the main cereal crops in the world, ranking fourth in terms of planted area and total production after maize, wheat and rice (FAOSTAT, 2016). It has very broad ecological adaptation and it is grown in regions with climates unfavorable for production of other cereals. It is commonly grown under dry conditions, poor and even saline soils. Due to these characteristics, it has been the principal grain produced in numerous stress-prone areas (Poehlman, 1985). The major uses of barley today are; mainly for livestock feed, malt, a component in a variety of foods, and has minor uses in the pharmaceutical industry (Biel and Jacyno, 2013). The barley cultivation area in the world in 2014 was 49.7 million hectares produced 8.5 million tons (FAOSTAT, 2016). While Egypt was involved in these values with low cultivation area which was 82500 hectares produced 5100 tons, therefore, it is needed to increase both; cultivated area and productivity.

Salinity in irrigation water and in soil is one of the major limiting factors for agricultural productivity. Large proportion of the total land area in the world is salt-affected. Egypt faces salinity problem and exploiting these areas is necessary to increase the production and reduce the gap between production and consumption of important crops (El - Hendawy et al., 2009). Therefore, using salt tolerant genotypes present an important solution for this economic problem (Kulshreshtha \& Singh, 2011).

Developing high yielding-varieties and tolerant to biotic or abiotic stresses is the main objective of breeding programs. It is necessary to have suitable information about the nature of genetic variability in the available breeding materials to achieve efficient selection (Zecevic et al., 2010). The selection mainly depends on additive genetic variance, effect of the environment and genotype by environment interaction (Eshghi \& Akhundova, 2009).

Diallel cross analysis is a useful mating system used by plant breeders and geneticists to estimate the genetic nature of qualitative and quantitative traits (Hallauer \& Miranda, 1988). It is an effective analysis to compute general (GCA) and specific combining ability (SCA) in a set of genotypes and compare their performance in different crosses combinations, and also to investigate the action of genes and heritability of important traits (Walejkl \& Rusell, 1977; Salgotra et al., 2009 and Zhang et al., 2015). It leads to identify suitable parents with high combining ability, which is very useful for producing hybrids with greater

Egypt. J. Agron. Vol. 38, No. 3 (2016) 
heterosis in barley breeding programs (Zhang et al., 2015). The genotypes which present high GCA indicate that they have ability to combine well with others and could be used to produce improved lines in breeding programs as well as indicate to additive gene effects for the studied traits (Madić et al., 2014). On the other hand the genotypes which present high values of SCA indicate to combine well only in certain crosses also indicate to dominance gene effects for the studied traits (Qu et al., 2012). The combining ability can be analyzed using the Griffing (1956) method, whereas to study gene action, genetic components and heritability, the Hayman (1954) method can be used. Griffing and Hayman analyses are often used together for completing data interpretation (Syukur et al., 2010). This provides an opportunity to obtain a rapid and general picture of genetic control for a set of genotypes in early generations. Also the regression graph of covariance $(\mathrm{Wr})$ and variance $(\mathrm{Vr})$ of arrays in diallel crosses provided useful information for the average degree of dominance of genes affecting the studied traits and the distribution of dominant and recessive alleles in the used parents (Jinks \& Hayman, 1953 and Jana, 1975).

The aim of this work was to determine general and specific combining ability in a half diallel cross involving eight spring barley genotypes and their F1 crosses. And also to study the gene action and heritability of different agronomic traits under normal and salinity conditions, which provide useful information for barley breeding programs.

\section{Materials and Methods}

\section{Field experiments and plant materials}

The plant materials used in this study included four commercial cultivars and the others were introduced from ICARDA (Table 1). In the first season 20142015, parents were grown in the Experimental Farm of Faculty of Agriculture (Ghazala), Zagazig University, Egypt $\left(30^{\circ} 34^{\prime} \mathrm{N}, 31^{\circ} 34^{\prime} \mathrm{E}\right) .8 \times 8$ half diallel mating system was used to produce $28 \mathrm{~F} 1$ hybrids. In the second season 2015 2016 the derived hybrid seeds from 28 crosses and their parents were sown at two distinct locations. The first was in Ghazala as normal condition and the other was under salinity condition in the Experimental Farm of Desert Research Center, Ras-Sudr Research Station, South Sinai, Egypt (29 $\left.35^{\prime} \mathrm{N}, 32^{\circ} 41^{\prime} \mathrm{E}\right)$ with salinity in irrigation water and soil containing $4500 \mathrm{ppm}$ and $5535 \mathrm{ppm}$, respectively (Table 2). Both experiments were layout in the field of a randomized complete block design (RCBD) with three replications. Each of 36 entries was planted in a single row, 2 meter long with $20 \mathrm{~cm}$ between rows and $10 \mathrm{~cm}$ within rows spacing's. All recommended agronomic practices from sowing until harvesting including irrigation, nitrogen, potassium and phosphate fertilizers and pest, disease and weed control were applied for barley in each region.

\section{Studied traits}

Ten plants were selected randomly from each replication for parents and F1s to record observations of; plant height $(\mathrm{cm})$, spike length $(\mathrm{cm})$, number of spikes 
per plant, number of grains per spike, grain weight per spike (g), 100 grain weight $(\mathrm{g})$ and grain yield per plant $(\mathrm{g})$.

TABLE 1. Name, origin and pedigree of eight barley genotypes.

\begin{tabular}{|c|c|c|c|c|}
\hline Codes & Genotype & Origin & $\begin{array}{c}\text { Year } \\
\text { of } \\
\text { release }\end{array}$ & Pedigree \\
\hline P1 & Giza 123 & Egypt & 1988 & Giza 117 / FAO 86 \\
\hline $\mathrm{P} 2$ & Giza 126 & Egypt & 1995 & $\begin{array}{c}\text { Baladi Bahteem/S D729-Por12762- } \\
\text { BC }\end{array}$ \\
\hline P3 & Australian & ICARDA & - & $\begin{array}{l}\text { SALIAN/4/DEIRALLA106/CEL/3/ } \\
\text { BCOMR/MZG//APM/5106 }\end{array}$ \\
\hline P4 & CHK 38 & ICARDA & - & $\begin{array}{c}\text { BARBATA/4/BACA(S)/3/AE253// } \\
\text { EI08887/CIO5761/5/DAT00CRA }\end{array}$ \\
\hline P5 & CHK 2 & ICARDA & - & $\begin{array}{l}\text { MARI/ATHS*2//AVT/ATTIKI/3/ } \\
\text { ATHS /LIGNEE686/5/AGER//API/ } \\
\text { CM67 } \\
\text { /3/CEL/WI2269//ORE/4/ALANDA }\end{array}$ \\
\hline P6 & CHK 53 & ICARDA & - & $\begin{array}{l}\text { Atahualpa//Alanda- } \\
\text { 01/Hamra/3/Keel ICB03-0170- } \\
\text { 26AP-0AP }\end{array}$ \\
\hline P7 & Giza 132 & ICARDA & 2006 & $\begin{array}{c}\text { Rihane-O5 // AS } 46 \text { / Aths*2" Aths } \\
\text { / Lignee } 686\end{array}$ \\
\hline P8 & Giza 2000 & Egypt & 2000 & $\begin{array}{c}\text { Giza } 117 \text { / Bahtim } 52 \text { // Giza } 118 \text { / } \\
\text { FAO 86/3/ Baladi 16/ Gem. (Giza } \\
\text { 121) }\end{array}$ \\
\hline
\end{tabular}

Egypt. J. Agron. Vol. 38, No. 3 (2016) 
TABLE 2 Soil properties of the experimental sites Ghazala and Ras-Sudr.

\begin{tabular}{|c|c|c|c|}
\hline & $\begin{array}{c}\text { Ghazalah } \\
\text { (Soil) }\end{array}$ & $\begin{array}{c}\text { Ras-Sudr } \\
\text { (Soil) }\end{array}$ & $\begin{array}{c}\text { Ras-Sudr } \\
\text { (Irrigation } \\
\text { water) }\end{array}$ \\
\hline Sand $\%$ & 20.61 & 86.08 & \\
\hline Silt\% & 31.82 & 8.05 & \\
\hline Clay\% & 47.57 & 10.67 & \\
\hline Textural class & Clay & Sandy loam & \\
\hline $\mathrm{CaCO} 3\left(\mathrm{~g} \mathrm{~kg}^{-1}\right)$ & 6.14 & 56.99 & \\
\hline Organic matter $\left(\mathrm{g} \mathrm{kg}^{-1}\right)$ & 10.34 & 2.20 & \\
\hline $\mathrm{pH}$ & 8.02 & 7.78 & 8.62 \\
\hline $\mathrm{EC}\left(\mathrm{dSm}^{-1}\right)$ & 1.94 & 8.65 & 7.03 \\
\hline \multicolumn{4}{|c|}{ Soluble cations and anions $\left(\mathrm{mmolc}^{-1}\right)$} \\
\hline $\mathrm{Ca}^{++}$ & 5.22 & 38.22 & \\
\hline $\mathrm{Mg}^{++}$ & 4.37 & 27.44 & \\
\hline $\mathrm{Na}^{+}$ & 4.52 & 58.83 & 40.05 \\
\hline $\mathrm{K}^{+}$ & 5.39 & 2.01 & 0.12 \\
\hline $\mathrm{HCO}^{-}$ & 6.08 & 3.43 & 4.54 \\
\hline$\overline{\mathrm{Cl}^{-}}$ & 6.58 & 64.14 & 48.94 \\
\hline $\mathrm{SO} 4=$ & 6.84 & 58.93 & 29.23 \\
\hline \multicolumn{4}{|c|}{ Available nutrient (mg kg-1 soil ) } \\
\hline $\mathrm{N}$ & 57.32 & 20.20 & \\
\hline $\mathrm{P}$ & 8.15 & 4.10 & \\
\hline $\mathrm{K}$ & 149.3 & 50.80 & \\
\hline
\end{tabular}

Statistical analysis

Data obtained from barley Diallel crosses was statistically were performed according to (Griffing, 1956) method 2, model 1, the analysis was applied for each environment separately. Also, the components of genetic variance and $\mathrm{Wr}-\mathrm{Vr}$ graphs were estimated by Hayman (1954a), Hayman (1954b) and Mather \& Jinks (1982).

\section{Results and Discussion}

For generating useful information on general and specific combining ability, gene action and heritability for important agronomic traits, diallel analysis for eight diverse spring barley genotypes was performed. This could help in the selection process in breeding programs under normal and salinity conditions. 
Analysis of variance

Analysis of variance for studied traits is presented in Table 3. It was found highly significant differences between genotypes at both locations for all studied traits. This result provides evidence for presence of genetic variability in the used materials, which could be exploited in barley breeding programs for improving yield and its contributing traits. Dividing the genotypic variance into parents, crosses and parent vs. crosses, indicated that the variance due to parents as well as crosses were highly significant for all studied traits. Also it was found highly significant differences among parents and their F1 crosses for all traits except plant height and 100 grain weight under normal condition (Ghazala). Similar differences between parents and crosses were found by other researchers as Eshghi \& Akhundova (2009), Saad et al. (2013), Zhang et al. (2015), Ahmadi et al. (2016), Patial et al. (2016) and Pesaraklu et al. (2016).

The variance due to general (GCA) and specific combining ability (SCA) are showed in Table 3. It was found that the variance of GCA and SCA was highly significant under both conditions for all studied traits. This indicated that both additive and non-additive gene actions contributed significantly in the inheritance of these traits, and it reveals the importance of both types of gene action in selecting transgressive genotypes through barley breeding programs. However, the variance due to SCA was higher than that of GCA for all traits except plant height and number of spikes per plant under salinity condition and 100 grain weight under normal condition. This result suggested the importance of non-additive gene action in the inheritance of these traits. Furthermore the ratio of GCA/SCA was less than one supporting the forgoing result. Hence, selection for these traits should be in later generation within the segregating populations. The importance of additive and non-additive gene action in the inheritance of the studied traits was confirmed previously by different authors Yilmaz \& Konak (2000), Ali et al. (2007), Potla et al. (2013), Saad et al. (2013), Madić et al. (2014), Tofiq et al. (2015) and Patial et al. (2016).

\section{Mean performance of parents and F1 crosses}

Mean performance of barley genotypes and their F1 crosses for studied traits under both conditions are showed in Table 4. Wide significant differences were noticed among the parents and the crosses for all studied traits under both conditions. Also, it was observed considerable differences between the two locations of study for all traits. The mean values of traits reduced at Ras-Sudr (salinity condition) compared with their respective at Ghazala (normal condition). This reduction was caused by the salinity stress effect. The highest reduction was assigned for grain yield per plant $(37.8 \%)$ followed by number of spikes per plant $(28.2 \%)$ and plant height $(14.5 \%)$. Otherwise the lowest reduction was assigned for Spike length $(8.1 \%)$ followed by 100 grain weight $(10.1 \%)$, number of grains per spike $(12.3 \%)$ and grain weight per spike $(12.6 \%)$. 

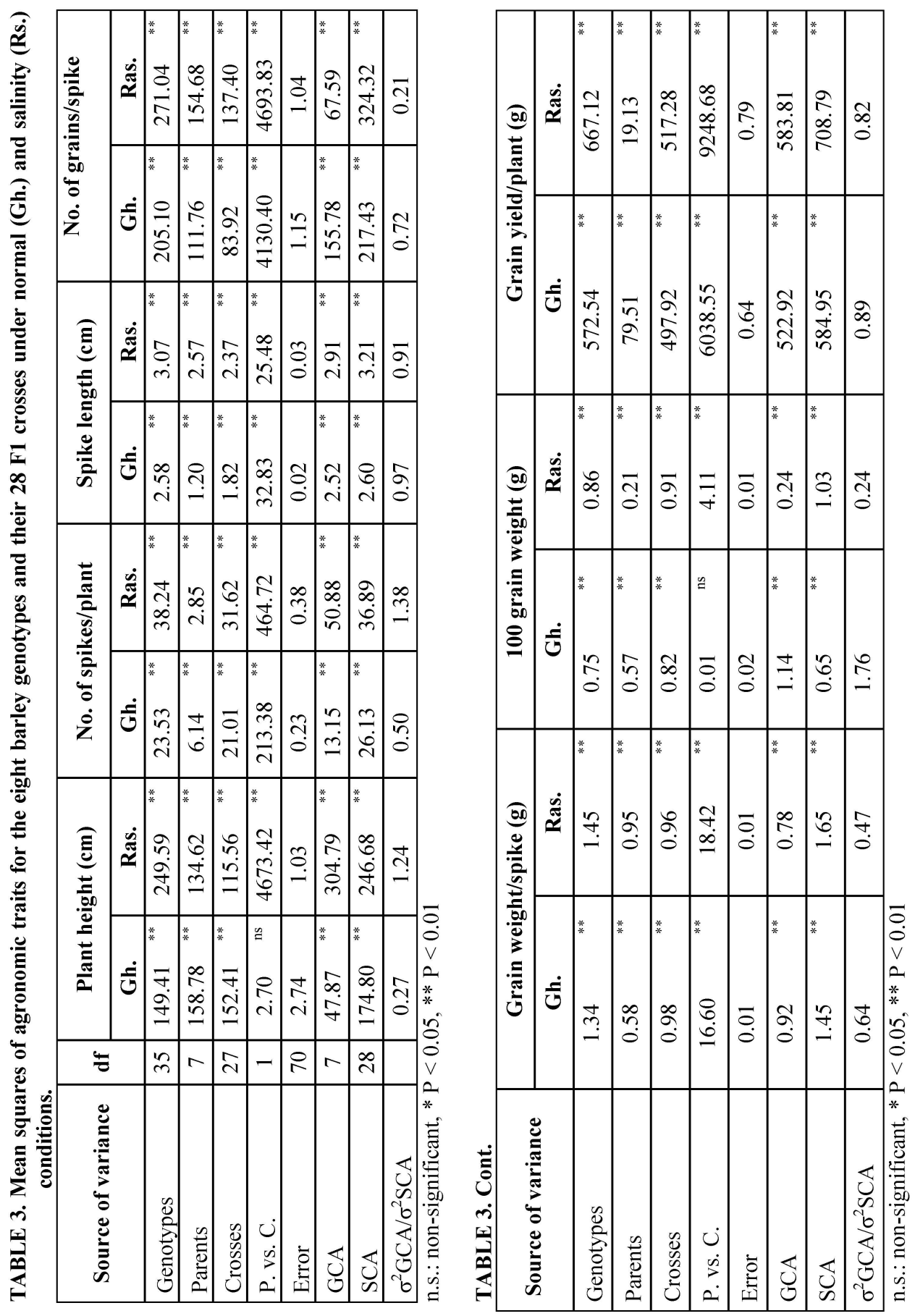

Egypt. J. Agron. Vol. 38, No. 3 (2016) 
TABLE 4. Mean performances of the agronomic traits for eight barley genotypes and their 28 F1 crosses under normal (Gh.) and salinity (Rs.) conditions.

\begin{tabular}{|c|c|c|c|c|c|c|}
\hline \multirow{2}{*}{ Genotypes } & \multicolumn{2}{|c|}{ Plant height (cm) } & \multicolumn{2}{|c|}{ No. of spikes/plant } & \multicolumn{2}{|c|}{ Spike length $(\mathrm{cm})$} \\
\hline & Gh. & Ras. & Gh. & Ras. & Gh. & Ras. \\
\hline P1 & 81.0 & 66.2 & 11.4 & 5.6 & 6.6 & 6.6 \\
\hline $\mathrm{P} 2$ & 93.9 & 68.9 & 10.9 & 6.0 & 6.7 & 6.2 \\
\hline P3 & 100.8 & 51.1 & 11.9 & 6.6 & 7.7 & 7.6 \\
\hline $\mathrm{P} 4$ & 95.6 & 65.4 & 8.4 & 6.2 & 6.1 & 4.8 \\
\hline P5 & 96.1 & 73.9 & 9.4 & 6.9 & 6.3 & 5.4 \\
\hline P6 & 81.4 & 65.9 & 10.4 & 7.1 & 6.4 & 6.2 \\
\hline P7 & 86.1 & 67.2 & 11.4 & 5.2 & 7.5 & 7.0 \\
\hline P8 & 93.1 & 60.6 & 13.0 & 4.2 & 6.0 & 5.4 \\
\hline $\mathrm{P} 1 \times \mathrm{P} 2$ & 91.1 & 79.8 & 12.4 & 8.6 & 8.5 & 7.4 \\
\hline $\mathrm{P} 1 \times \mathrm{P} 3$ & 104.0 & 74.3 & 14.9 & 10.1 & 7.8 & 7.4 \\
\hline $\mathrm{P} 1 \times \mathrm{P} 4$ & 91.0 & 76.9 & 13.4 & 6.1 & 7.9 & 7.8 \\
\hline $\mathrm{P} 1 \times \mathrm{P} 5$ & 95.7 & 77.4 & 13.4 & 8.4 & 7.9 & 7.5 \\
\hline $\mathrm{P} 1 \times \mathrm{P} 6$ & 80.5 & 79.7 & 13.9 & 6.7 & 7.8 & 7.5 \\
\hline $\mathrm{P} 1 \times \mathrm{P} 7$ & 90.4 & 84.7 & 11.7 & 11.7 & 8.6 & 5.8 \\
\hline $\mathrm{P} 1 \times \mathrm{P} 8$ & 85.2 & 84.3 & 13.4 & 6.7 & 7.7 & 7.4 \\
\hline $\mathrm{P} 2 \times \mathrm{P} 3$ & 80.2 & 80.1 & 14.7 & 14.6 & 7.7 & 7.1 \\
\hline $\mathrm{P} 2 \times \mathrm{P} 4$ & 85.2 & 73.7 & 12.4 & 10.4 & 7.3 & 7.2 \\
\hline $\mathrm{P} 2 \times \mathrm{P} 5$ & 75.9 & 71.8 & 11.3 & 9.8 & 6.3 & 6.1 \\
\hline $\mathrm{P} 2 \times \mathrm{P} 6$ & 95.2 & 68.2 & 14.4 & 9.2 & 8.4 & 6.2 \\
\hline $\mathrm{P} 2 \times \mathrm{P} 7$ & 99.8 & 76.2 & 12.4 & 9.8 & 9.2 & 8.6 \\
\hline $\mathrm{P} 2 \times \mathrm{P} 8$ & 84.3 & 81.8 & 12.9 & 7.2 & 7.6 & 6.5 \\
\hline $\mathrm{P} 3 \times \mathrm{P} 4$ & 95.6 & 79.8 & 18.4 & 9.8 & 8.3 & 7.6 \\
\hline $\mathrm{P} 3 \times \mathrm{P} 5$ & 91.0 & 80.3 & 14.7 & 14.6 & 8.1 & 7.7 \\
\hline $\mathrm{P} 3 \times \mathrm{P} 6$ & 87.9 & 74.8 & 11.7 & 11.6 & 7.8 & 7.4 \\
\hline $\mathrm{P} 3 \times \mathrm{P} 7$ & 80.4 & 73.2 & 13.3 & 12.8 & 6.6 & 5.9 \\
\hline $\mathrm{P} 3 \times \mathrm{P} 8$ & 83.4 & 79.2 & 11.3 & 10.2 & 8.7 & 8.4 \\
\hline $\mathrm{P} 4 \times \mathrm{P} 5$ & 91.0 & 87.8 & 16.3 & 7.6 & 6.5 & 6.3 \\
\hline $\mathrm{P} 4 \times \mathrm{P} 6$ & 93.6 & 79.9 & 14.4 & 12.8 & 7.6 & 6.2 \\
\hline $\mathrm{P} 4 \times \mathrm{P} 7$ & 90.2 & 84.9 & 17.3 & 9.7 & 9.0 & 8.5 \\
\hline $\mathrm{P} 4 \times \mathrm{P} 8$ & 90.7 & 82.3 & 19.3 & 9.8 & 7.6 & 7.4 \\
\hline $\mathrm{P} 5 \times \mathrm{P} 6$ & 90.1 & 89.9 & 15.1 & 15.0 & 7.9 & 7.1 \\
\hline $\mathrm{P} 5 \times \mathrm{P} 7$ & 98.7 & 94.3 & 19.3 & 16.2 & 9.5 & 9.3 \\
\hline $\mathrm{P} 5 \times$ P8 & 99.0 & 87.2 & 18.9 & 18.8 & 8.1 & 7.4 \\
\hline $\mathrm{P} 6 \times \mathrm{P} 7$ & 95.7 & 88.8 & 19.3 & 15.3 & 8.7 & 8.2 \\
\hline $\mathrm{P} 6 \times \mathrm{P} 8$ & 103.7 & 78.6 & 11.3 & 9.2 & 7.4 & 6.6 \\
\hline $\mathrm{P} 7 \times \mathrm{P} 8$ & 90.1 & 89.9 & 14.6 & 14.4 & 8.9 & 8.5 \\
\hline L.S.D ${ }_{0.05}$ & 2.69 & 1.66 & 0.78 & 1.00 & 0.20 & 0.30 \\
\hline
\end{tabular}

It was noticed that the parent Giza 126 (P2) showed the highest values for number of grains per spike, 100 grain weight, grain weight per spike and grain yield under both conditions. Also, Australian (P3) exhibited highest values for spike length under both conditions, as well as presented highest plant height under normal condition; while CHK 2 (P5) surpassed the other genotypes under salinity condition. The shortest plant height was assigned for CHK 53 (P6) under normal condition and Australian (P3) under salinity condition. CHK 53(P6) gave the highest number of spikes per plant under salinity condition. Otherwise, Giza 2000 (P8) was the highest one under normal condition. Thus, these genotypes could be considered as good genotypes for increasing grain yield and its attributes under the target environment.

Also it was observed that the F1 crosses showed performance better than the parents in all studied traits (Table 4). The cross, $\mathrm{P} 5 \times \mathrm{P} 7$ presented the tallest plant height under salinity condition, and spike length under both conditions and highest

Egypt. J. Agron. Vol. 38, No. 3 (2016) 
grain yield per plant under normal condition. Barley cross $\mathrm{P} 6 \times \mathrm{P} 7$ produced the highest number of spikes per plant under normal condition and number of grains per spike under salinity condition. Otherwise $\mathrm{P} 5 \times \mathrm{P} 8$ surpassed the other crosses in number of spikes per plant and grain yield per plant under salinity condition. $\mathrm{P} 2 \times \mathrm{P} 7$ produced the greatest number of grains per spike under normal condition and 100 grain weight under salinity one. Furthermore $\mathrm{P} 7 \times \mathrm{P} 8$ exhibited the highest grain weight per spike under both conditions. Similar genetic differences for studied traits were previously found by Ahmed et al. (1998), Sharma et al. (2002), Ali et al. (2007), Eshghi \& Akhundova (2009), Aghamiri et al. (2012), Saad et al. (2013), Zhang et al. (2015), Patial et al. (2016) and Pesaraklu et al. (2016).

TABLE 4. Cont.

\begin{tabular}{|c|c|c|c|c|c|c|c|c|}
\hline \multirow[t]{2}{*}{ Genotypes } & \multicolumn{2}{|c|}{$\begin{array}{c}\text { No. of } \\
\text { grains/spike }\end{array}$} & \multicolumn{2}{|c|}{$\begin{array}{c}\text { Grain } \\
\text { weight/spike (g) }\end{array}$} & \multicolumn{2}{|c|}{$\begin{array}{c}100 \text { grain weight } \\
(\mathrm{g})\end{array}$} & \multicolumn{2}{|c|}{$\begin{array}{c}\text { Grain yield/plant } \\
(\mathrm{g})\end{array}$} \\
\hline & Gh. & Ras. & Gh. & Ras. & Gh. & Ras. & Gh. & Ras. \\
\hline P1 & 48.1 & 45.2 & 2.9 & 2.7 & 5.7 & 4.8 & 29.9 & 10.6 \\
\hline $\mathrm{P} 2$ & 57.3 & 52.2 & 3.5 & 3.3 & 6.6 & 5.3 & 34.0 & 13.7 \\
\hline P3 & 43.7 & 33.1 & 2.5 & 1.9 & 5.7 & 4.4 & 28.5 & 8.3 \\
\hline $\mathrm{P} 4$ & 40.7 & 30.7 & 2.3 & 1.5 & 5.8 & 5.0 & 18.9 & 8.5 \\
\hline P5 & 47.4 & 36.9 & 2.6 & 2.3 & 6.2 & 4.8 & 28.1 & 10.5 \\
\hline P6 & 41.0 & 40.7 & 2.3 & 2.1 & 5.3 & 4.6 & 21.5 & 10.5 \\
\hline P7 & 51.9 & 33.9 & 3.1 & 2.3 & 6.1 & 4.8 & 32.2 & 7.0 \\
\hline P8 & 40.1 & 35.3 & 2.3 & 1.8 & 5.9 & 5.0 & 29.8 & 5.6 \\
\hline $\mathrm{P} 1 \times \mathrm{P} 2$ & 70.8 & 54.7 & 3.8 & 2.9 & 5.1 & 4.9 & 42.6 & 21.7 \\
\hline $\mathrm{P} 1 \times \mathrm{P} 3$ & 54.2 & 51.0 & 4.0 & 3.5 & 6.0 & 5.6 & 44.0 & 29.3 \\
\hline $\mathrm{P} 1 \times \mathrm{P} 4$ & 56.7 & 55.2 & 3.2 & 3.2 & 7.0 & 5.4 & 51.0 & 18.4 \\
\hline $\mathrm{P} 1 \times \mathrm{P} 5$ & 67.0 & 55.7 & 4.2 & 4.0 & 5.6 & 5.3 & 48.5 & 25.7 \\
\hline $\mathrm{P} 1 \times \mathrm{P} 6$ & 57.6 & 53.3 & 3.4 & 3.1 & 6.2 & 5.7 & 24.7 & 20.4 \\
\hline $\mathrm{P} 1 \times \mathrm{P} 7$ & 55.2 & 36.6 & 2.7 & 2.5 & 6.2 & 5.6 & 29.7 & 22.1 \\
\hline $\mathrm{P} 1 \times \mathrm{P} 8$ & 55.6 & 53.3 & 3.6 & 3.4 & 5.4 & 5.3 & 34.9 & 16.2 \\
\hline $\mathrm{P} 2 \times \mathrm{P} 3$ & 55.5 & 47.8 & 3.0 & 2.3 & 5.4 & 5.1 & 35.4 & 34.1 \\
\hline $\mathrm{P} 2 \times \mathrm{P} 4$ & 61.1 & 60.3 & 3.8 & 3.4 & 5.6 & 5.2 & 34.2 & 31.9 \\
\hline $\mathrm{P} 2 \times \mathrm{P} 5$ & 59.9 & 57.4 & 3.6 & 3.2 & 5.1 & 5.1 & 32.8 & 29.5 \\
\hline $\mathrm{P} 2 \times \mathrm{P} 6$ & 66.6 & 47.9 & 2.8 & 2.6 & 5.2 & 4.9 & 47.1 & 21.0 \\
\hline $\mathrm{P} 2 \times \mathrm{P} 7$ & 71.2 & 51.7 & 4.1 & 3.8 & 6.5 & 6.4 & 55.7 & 29.8 \\
\hline $\mathrm{P} 2 \times \mathrm{P} 8$ & 56.7 & 55.2 & 3.0 & 2.4 & 6.0 & 5.6 & 31.0 & 21.4 \\
\hline $\mathrm{P} 3 \times \mathrm{P} 4$ & 61.5 & 59.4 & 4.3 & 4.1 & 5.8 & 5.4 & 62.8 & 35.6 \\
\hline $\mathrm{P} 3 \times \mathrm{P} 5$ & 64.1 & 61.2 & 4.2 & 3.6 & 6.4 & 6.2 & 54.3 & 52.8 \\
\hline $\mathrm{P} 3 \times \mathrm{P} 6$ & 64.0 & 62.2 & 3.5 & 3.3 & 5.5 & 5.3 & 44.0 & 40.7 \\
\hline $\mathrm{P} 3 \times \mathrm{P} 7$ & 56.6 & 53.3 & 3.5 & 3.0 & 5.4 & 3.7 & 38.2 & 24.7 \\
\hline $\mathrm{P} 3 \times \mathrm{P} 8$ & 67.2 & 64.6 & 3.5 & 3.3 & 5.2 & 5.0 & 37.2 & 35.4 \\
\hline $\mathrm{P} 4 \times \mathrm{P} 5$ & 60.5 & 57.7 & 4.0 & 3.3 & 5.2 & 4.6 & 44.5 & 14.6 \\
\hline $\mathrm{P} 4 \times \mathrm{P} 6$ & 60.2 & 53.1 & 3.0 & 2.6 & 5.7 & 5.5 & 44.1 & 41.0 \\
\hline $\mathrm{P} 4 \times \mathrm{P} 7$ & 62.1 & 52.4 & 3.5 & 3.1 & 6.4 & 5.6 & 65.8 & 26.6 \\
\hline $\mathrm{P} 4 \times \mathrm{P} 8$ & 58.6 & 50.9 & 3.4 & 3.3 & 5.4 & 4.4 & 57.4 & 17.7 \\
\hline P5 $\times$ P6 & 59.2 & 54.7 & 3.5 & 3.0 & 5.8 & 5.5 & 42.3 & 40.7 \\
\hline $\mathrm{P} 5 \times \mathrm{P} 7$ & 67.4 & 60.8 & 4.0 & 3.8 & 6.3 & 5.2 & 78.5 & 51.6 \\
\hline P5 $\times$ P8 & 56.1 & 50.7 & 4.2 & 3.6 & 6.3 & 6.1 & 63.4 & 59.9 \\
\hline $\mathrm{P} 6 \times \mathrm{P} 7$ & 69.9 & 66.9 & 4.6 & 4.0 & 5.3 & 5.1 & 57.1 & 55.1 \\
\hline $\mathrm{P} 6 \times \mathrm{P} 8$ & 53.2 & 37.6 & 2.4 & 2.0 & 5.5 & 5.0 & 29.5 & 15.7 \\
\hline $\mathrm{P} 7 \times \mathrm{P} 8$ & 63.7 & 56.1 & 4.7 & 4.2 & 6.5 & 6.0 & 53.7 & 50.8 \\
\hline L.S.D ${ }_{0.05}$ & 1.75 & 1.66 & 0.15 & 0.18 & 0.22 & 0.16 & 1.31 & 1.45 \\
\hline
\end{tabular}


General and specific combining ability effects

The GCA effect was estimated of the parental genotypes for studied traits to identify the best parents and good combiners for producing transgressive phenotypes (Table 5). Negative GCA effect is desirable for plant height to avoid plant lodging, while for other traits positive effects are desirable to increase grain yield and its components. It was found that Giza 123 (P1) presented good combining ability for reducing plant height, furthermore it was a good combiner for increasing 100 grain weight under both conditions. Giza126 (P2) was a good combiner for reducing plant height and increasing number of grains per spike under both conditions. Australian (P3) was a good combiner for reducing plant height and increasing number of spikes plant, spike length, number of grains per spike and grain yield per plant under salinity condition. CHK 38 (P4) was a good combiner for number of spikes per plant and grain yield per plant under normal condition, otherwise, CHK 53 (P6) exhibited good combination for the same traits under salinity condition. CHK 2 (P5) and Giza 132 (P7) showed good combinations for grain yield and its components under both conditions. Giza 2000 (P8) was a good combiner for number of spikes per plant under normal condition and for 100 grain weight under salinity one. Hence, these genotypes can be further used in developing segregating populations in barley breeding programs, especially P3 and P6 under salinity condition, P4 and P8 under normal condition, and P5 and P7 under both conditions. Similar results in respect to GCA effects have been reported by Ali et al. (2007), Potla et al. (2013), Saad et al. (2013), Madić et al. (2014), Patial et al. (2016) and Pesaraklu et al. (2016).

TABLE 5. Estimates of general combining ability (GCA) effects of eight barley genotypes for the studied traits under normal (Gh.) and salinity (Rs.) conditions.

\begin{tabular}{|c|c|c|c|c|c|c|c|c|}
\hline \multirow{2}{*}{ Parents } & \multicolumn{2}{|c|}{ Plant height (cm) } & \multicolumn{2}{|c|}{ No. of spikes/plant } & \multicolumn{2}{|c|}{ Spike length (cm) } & \multicolumn{2}{|c|}{ No. of grains/spike } \\
\hline & Gh. & Ras. & Gh. & Ras. & Gh. & Ras. & Gh. & Ras. \\
\hline P1 & $-1.64^{* *}$ & $-0.53^{*}$ & $-0.57^{* *}$ & $-1.93^{* *}$ & $0.03 \quad$ ns & $0.03 \quad$ ns & $-0.74^{* *}$ & $-0.73^{* *}$ \\
\hline $\mathrm{P} 2$ & $-1.69^{* *}$ & $-2.53^{* *}$ & $-0.94^{\text {** }}$ & $-0.72^{* *}$ & $-0.09^{* *}$ & $-0.20^{* *}$ & $3.58^{* *}$ & 2.20 *** \\
\hline P3 & $0.77^{*}$ & $-5.07^{* *}$ & -0.12 ns & $0.84^{* *}$ & $0.11^{* *}$ & $0.32^{* *}$ & $-1.01^{* *}$ & $0.84^{* *}$ \\
\hline P4 & $1.20^{* *}$ & 0.12 ns & $0.71^{* *}$ & $-1.02^{* * *}$ & $-0.29^{* *}$ & $-0.31^{* * *}$ & $-1.85^{* *}$ & $-0.69^{* * *}$ \\
\hline P5 & $1.72^{* *}$ & $4.17^{* *}$ & $0.00^{\text {ns }}$ & $1.54^{* *}$ & $-0.22^{* *}$ & $-0.12^{* *}$ & $0.83^{* *}$ & $1.46^{* * *}$ \\
\hline P6 & $-0.68 \quad$ ns & -0.33 ns & $-0.50^{* *}$ & $0.54^{* *}$ & $-0.08^{*}$ & $-0.21^{* * *}$ & $-0.80^{* *}$ & $-0.04^{\mathrm{ns}}$ \\
\hline P7 & $-0.10^{n s}$ & $3.18^{* *}$ & $0.92^{* *}$ & $1.16^{* *}$ & $0.64^{* *}$ & $0.53^{* *}$ & $2.93^{* *}$ & $-1.19^{* *}$ \\
\hline P8 & $0.41^{\mathrm{ns}}$ & $0.98^{* *}$ & $0.50^{* *}$ & $-0.41^{* *}$ & $-0.11^{* *}$ & $-0.05^{\mathrm{ns}}$ & $-2.94^{* *}$ & $-1.85^{* * *}$ \\
\hline S.E.(gi-gj) & 0.282 & 0.174 & 0.082 & 0.105 & 0.021 & 0.031 & 0.183 & 0.174 \\
\hline
\end{tabular}

n.s.: non-significant, $* \mathrm{P}<0.05, * * \mathrm{P}<0.01$

Egypt. J. Agron. Vol. 38, No. 3 (2016) 
TABLE 5. Cont.

\begin{tabular}{|c|c|c|c|c|c|c|c|c|c|c|c|c|}
\hline \multirow{2}{*}{ Parents } & \multicolumn{4}{|c|}{ Grain weight/spike (g) } & \multicolumn{4}{|c|}{100 grain weight $(\mathrm{g})$} & \multicolumn{4}{|c|}{ Grain yield/plant (g) } \\
\hline & Gl & & $\mathbf{R a}$ & & Gl & & $\mathbf{R a}$ & & Gh & & Ra: & \\
\hline $\mathrm{P} 1$ & -0.01 & ns & 0.09 & $* *$ & 0.09 & $*$ & 0.05 & ** & -4.16 & $* *$ & -6.46 & $* *$ \\
\hline $\mathrm{P} 2$ & 0.04 & $*$ & 0.02 & ns & -0.26 & ** & 0.04 & $*$ & -3.35 & $* *$ & -2.29 & $* *$ \\
\hline $\mathrm{P} 3$ & 0.02 & $\mathrm{~ns}$ & -0.02 & $\mathrm{~ns}$ & -0.11 & ** & -0.18 & ** & -0.39 & $*$ & 2.94 & $* *$ \\
\hline $\mathrm{P} 4$ & -0.09 & ** & -0.11 & $* *$ & 0.05 & ns & -0.02 & ns & 2.08 & $* *$ & -3.71 & $* *$ \\
\hline P5 & 0.21 & ** & 0.19 & $* *$ & 0.19 & ** & 0.06 & ** & 4.36 & $* *$ & 5.60 & $* *$ \\
\hline P6 & -0.29 & ** & -0.22 & $* *$ & -0.24 & ** & -0.06 & $*$ & -4.51 & $* *$ & 1.58 & $* *$ \\
\hline $\mathrm{P} 7$ & 0.25 & ** & 0.18 & $* *$ & 0.29 & ** & 0.04 & $*$ & 6.97 & $* *$ & 3.49 & $* *$ \\
\hline P8 & -0.12 & ** & -0.12 & ** & 0.00 & ns & 0.06 & $*$ & -1.01 & ** & -1.15 & $* *$ \\
\hline S.E.(gi-gj) & 0.015 & & 0.018 & & 0.023 & & 0.017 & & 0.137 & & 0.152 & \\
\hline
\end{tabular}

N.S.: non-significant, $* \mathrm{P}<0.05,{ }^{* *} \mathrm{P}<0.01$

SCA effect is used for determining the best cross combinations for exploiting the heterosis. It was found significant effects for several crosses in each trait, indicates to presence of non-additive (dominance and epitasis) gene action (Table 6). The crosses; $\mathrm{P} 2 \times \mathrm{P} 5, \mathrm{P} 3 \times \mathrm{P} 7$ and $\mathrm{P} 2 \times \mathrm{P} 6$ exhibited the highest significant negative SCA effects for plant height under both conditions. Highest significant SCA values for number of spikes per plant and grain weight per spike were recorded by cross $\mathrm{P} 6 \times \mathrm{P} 7$ under normal condition as well as high values under salinity one. While under salinity condition the highest SCA for number of spikes per plant was recorded by $\mathrm{P} 5 \times \mathrm{P} 8$ and for grain weight per spike was recorded by $\mathrm{P} 3 \times \mathrm{P} 4$. Also $\mathrm{P} 6 \times \mathrm{P} 7$ presented the highest significant SCA for number of grains per spike under salinity condition as high value under normal condition. Otherwise, the highest one under normal condition was assigned for $\mathrm{P} 3 \times \mathrm{P} 8$ which exhibited also high value under salinity condition. The cross $\mathrm{P} 5 \times \mathrm{P} 7$ showed the highest SCA for spike length under both conditions. The highest SCA value of 100 grain weight was recorded by $\mathrm{P} 1 \times \mathrm{P} 4$ under normal condition while the highest one under salinity condition was $\mathrm{P} 2 \times \mathrm{P} 7$. Highest $\mathrm{SCA}$ value of grain yield per plant was assigned for $\mathrm{P} 5 \times \mathrm{P} 7$ under normal condition while the highest one under salinity condition was $\mathrm{P} 5 \times \mathrm{P} 8$. These cross combinations participated in at least one of good general combiner parent. Different crosses registered desirable SCA effects when studied by other authors (Ali et al., 2007; Potla et al., 2013; Saad et al., 2013; Madić et al., 2014; Patial et al., 2016 and Pesaraklu et al., 2016). 
TABLE 6. Estimates of specific combining ability (SCA) effects of the 28 crosses for the studied traits under normal (Gh.) and salinity (Rs.) conditions .

\begin{tabular}{|c|c|c|c|c|c|c|c|c|c|c|c|c|c|}
\hline \multirow{3}{*}{$\begin{array}{l}\text { Crosses } \\
\mathrm{P} 1 \times \mathrm{P} 2 \\
\end{array}$} & \multicolumn{4}{|c|}{ Plant height (cm) } & \multicolumn{3}{|c|}{ No. of spike/plant } & \multicolumn{3}{|c|}{ Spike length $(\mathrm{cm})$} & \multicolumn{3}{|c|}{ No. of grains/spike } \\
\hline & \multicolumn{2}{|l|}{ Gh. } & \multicolumn{2}{|l|}{ Ras. } & \multicolumn{2}{|l|}{ Gh. } & Ras. & \multicolumn{2}{|l|}{ Gh. } & Ras. & Gh. & \multicolumn{2}{|l|}{ Ras. } \\
\hline & 3.73 & & 5.63 & $* *$ & $0.45^{\text {ns }}$ & ns & $1.34^{* *}$ & $0.90^{*}$ & $* *$ & $.52^{* *}$ & $10.08^{* *}$ & 2.37 & *** \\
\hline$\times \mathrm{P} 3$ & 14.17 & $* *$ & 2.72 & $* *$ & $2.07^{* *}$ & $* *$ & $1.33^{* *}$ & $0.00^{\mathrm{n}}$ & ns & $.04 \mathrm{~ns}$ & $-1.93^{* *}$ & 0.07 & $\mathrm{~ns}$ \\
\hline$\times \mathrm{P} 4$ & 73 & ns & 0.09 & ns & $-0.21^{\mathrm{ns}}$ & ns & $-0.80^{*}$ & $0.42^{*}$ & ** & $0.99^{* *}$ & 1.43 & 5.82 & ${ }^{* *}$ \\
\hline$\times \mathrm{P} 5$ & 4.88 & $* *$ & -3.41 & $* *$ & 0.50 ns & ns & $-1.03^{* *}$ & $0.41^{*}$ & $* *$ & $0.55^{* *}$ & $9.07^{* *}$ & 4.12 & $* *$ \\
\hline $\mathrm{P} 1 \times \mathrm{P} 6$ & -7.86 & $* *$ & 3.32 & $* *$ & $1.45^{* *}$ & $* *$ & $-1.81^{* *}$ & 0.17 * & * & $0.57^{* *}$ & 1.24 ns & 3.28 & $* *$ \\
\hline$\times \mathrm{P} 7$ & 48 & ns & 4.81 & $* *$ & $-2.41^{* *}$ & $* *$ & $2.58^{* *}$ & $0.27^{*}$ & $* *$ & $-1.84^{* *}$ & $-4.82^{* *}$ & $*-12.35$ & ** \\
\hline P8 & 26 & $* *$ & 6.68 & $* *$ & $0.01 \quad$ ns & ns & $-0.85^{*}$ & $0.11^{\mathrm{n}}$ & ns & $.32^{*}$ & $1.46^{*}$ & 5.09 & ** \\
\hline $\mathrm{P} 2 \times \mathrm{P} 3$ & 68 & ** & 10.72 & *** & $1.89^{* *}$ & $* *$ & $4.68^{* *}$ & $-0.03^{n}$ & ns & $.07 \mathrm{~ns}$ & $-4.89^{* *}$ & -6.08 & $* *$ \\
\hline $\mathrm{P} 2 \times \mathrm{P} 4$ & -5.00 & $* *$ & -1.13 & ns & $-0.83^{* *}$ & $* *$ & $2.32^{* *}$ & $-0.04^{n}$ & ns & $0.61^{* *}$ & $1.53 *$ & 8.01 & ** \\
\hline $\mathrm{P} 2 \times \mathrm{P} 5$ & 4.85 & $* *$ & -7.07 & $* *$ & $-1.23^{* *}$ & $* *$ & $-0.91^{*}$ & $-1.10^{*}$ & $* *$ & $-0.61^{* *}$ & $-2.31^{* *}$ & 2.96 & ** \\
\hline $\mathrm{P} 2 \times \mathrm{P} 6$ & 88 & $* *$ & -6.13 & $* *$ & $2.38^{* *}$ & ${ }^{* *}$ & $-0.50 \quad \mathrm{~ns}$ & $0.83^{*}$ & $* *$ & $-0.43^{* *}$ & $5.97^{* *}$ & $\begin{array}{l}* \\
*\end{array}$ & $* *$ \\
\hline $\mathrm{P} 2 \times \mathrm{P} 7$ & 10.86 & $* *$ & -1.63 & $*$ & $-1.04^{* *}$ & $* *$ & $-0.53 \quad \mathrm{~ns}$ & $0.96^{*}$ & $* *$ & $1.20^{* *}$ & $6.84^{* *}$ & -0.16 & $\mathrm{~ns}$ \\
\hline $\mathrm{P} 2 \times \mathrm{P} 8$ & -5.09 & $* *$ & 6.12 & $* *$ & -0.17 ns & ns & $-1.51^{* *}$ & $0.13^{\mathrm{n}}$ & ns & $-0.29^{*}$ & $-1.82^{*}$ & 4.05 & ** \\
\hline $\mathrm{P} 3 \times \mathrm{P} 4$ & 2.88 & ${ }^{*}$ & 7.51 & $* *$ & $35^{* *}$ & $* *$ & 9 ns & $4^{*}$ & $* *$ & $54^{* *}$ & $48^{* *}$ & 8 & $* *$ \\
\hline 1. & (7. & * & 4.02 & $* *$ & -0.05 ns & ns & $42^{* *}$ & $0.50^{*}$ & *** & $.48^{* *}$ & $6.41^{* *}$ & .10 & ${ }^{* *}$ \\
\hline$\times \mathrm{P} 6$ & 90 & ${ }^{*}$ & 2.96 & $* *$ & $-2.00^{* *}$ & $* *$ & $47 \quad \mathrm{~ns}$ & $05^{n}$ & ns & $.26^{*}$ & $7.96^{* *}$ & 10.60 & ** \\
\hline $\mathrm{P} 3 \times \mathrm{P} 7$ & 0.92 & $* *$ & -2.10 & $* *$ & $-0.97^{* *}$ & $* *$ & $0.91 \quad \mathrm{~ns}$ & $-1.86^{*}$ & ** & $-1.97^{* *}$ & $-3.13^{* *}$ & 2.86 & ** \\
\hline $\mathrm{P}_{3}$ & .44 & $* *$ & 6.10 & $* *$ & $-2.55^{* *}$ & $* *$ & $-0.07 \quad$ ns & $05^{*}$ & ** & $1.03^{* *}$ & $13.30^{* *}$ & 14.75 & $* *$ \\
\hline $\mathrm{P} 4 \times \mathrm{P} 5$ & -2.63 & ${ }^{*}$ & 6.27 & $* *$ & $2.11^{* * *}$ & ${ }^{* *}$ & $-2.83^{* *}$ & $-0.65^{*}$ & ** & $-0.36^{* *}$ & $3.67^{\text {** }}$ & 6.08 & ** \\
\hline $\mathrm{P} 4 \times \mathrm{P} 6$ & 2.37 & * & 2.89 & $* *$ & $0.73^{*}$ & * & $3.39^{* *}$ & $0.25^{*}$ & ** & $-0.36^{* *}$ & $4.98^{* *}$ & 3.02 & ** \\
\hline $\mathrm{P} 4 \times \mathrm{P} 7$ & -1.59 & ns & 4.38 & $* *$ & $2.20^{* *}$ & $* *$ & $-0.33^{\mathrm{ns}}$ & $0.97^{*}$ & ** & $.21^{* *}$ & $3.21^{* *}$ & 3.50 & $* *$ \\
\hline $\mathrm{P} 4 \times \mathrm{P} 8$ & -1.63 & ns & 4.02 & $* *$ & $4.62^{* *}$ & ** & $1.35^{* *}$ & $0.31^{*}$ & ** & $0.74^{* *}$ & $5.57^{* *}$ & 2.61 & ** \\
\hline & -1.04 & ns & 8.89 & $* *$ & $-1.67^{* *}$ & $* *$ & $3.05^{* *}$ & $0.55^{*}$ & *** & $0.39^{* *}$ & 1.30 ns & 2.42 & $* *$ \\
\hline & ד ד ד & 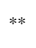 & 9.77 & $* *$ & $4.91^{* *}$ & $* *$ & $3.66^{* *}$ & $1.42^{*}$ & ** & $1.80^{\text {** }}$ & $5.80^{* *}$ & 9.68 & $* *$ \\
\hline $\mathrm{P} 5 \times \mathrm{P} 8$ & 0.10 & $* *$ & 4.86 & $* *$ & $3.55^{* *}$ & $* *$ & $7.79^{* *}$ & $0.78^{*}$ & $* *$ & $0.52^{* *}$ & 0.33 ns & 0.23 & $\mathrm{~ns}$ \\
\hline $\mathrm{P} 6$ & 1 & $* * *$ & 8.72 & ** & $5.41^{* *}$ & $* *$ & $3.77^{* *}$ & $0.48^{*}$ & ** & $0.78^{* *}$ & $9.91^{* *}$ & 17.29 & 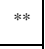 \\
\hline $\mathrm{P} 6 \times \mathrm{P} 8$ & 13.23 & ** & 0.70 & ns & $-2.17^{* *}$ & $* *$ & $-0.77^{\mathrm{ns}}$ & $-0.06^{n}$ & ns & $-0.26^{*}$ & -0.92 ns & \begin{tabular}{l|l} 
s & -11.38 \\
\end{tabular} & ${ }^{* *}$ \\
\hline $\mathrm{P} 7 \times \mathrm{P} 8$ & -3.13 & & 8.74 & *** & -0.31 ns & ns & $3.84^{* *}$ & $0.68^{*}$ & *** & $0.99^{* *}$ & $5.86^{* *}$ & 8.33 & \\
\hline S.E.(sij - sj) & 1.05 & & 0.64 & & 0.30 & & 0.39 & 0.08 & & 0.12 & 0.68 & 0.64 & \\
\hline
\end{tabular}


TABLE 6. Cont.

\begin{tabular}{|c|c|c|c|c|c|c|c|c|c|c|c|c|}
\hline \multicolumn{13}{|c|}{ BLE 6. Cont. } \\
\hline \multirow{3}{*}{$\begin{array}{l}\text { Crosses } \\
\mathrm{P} 1 \times \mathrm{P} 2 \\
\end{array}$} & \multicolumn{4}{|c|}{ Grain weight/spike (g) } & \multicolumn{4}{|c|}{100 grain weight $(\mathrm{g})$} & \multicolumn{4}{|c|}{ Grain yield/plant (g) } \\
\hline & \multicolumn{2}{|c|}{ Gh. } & \multicolumn{2}{|c|}{ Ras. } & \multicolumn{2}{|c|}{ Gh. } & \multicolumn{2}{|c|}{ Ras. } & \multicolumn{2}{|c|}{ Gh. } & \multicolumn{2}{|l|}{ Ras. } \\
\hline & 0.34 & $* *$ & -0.19 & $*$ & -0.55 & $* *$ & -0.40 & $* *$ & 8.28 & $* *$ & 3.85 & $* *$ \\
\hline $\mathrm{P} 1 \times \mathrm{P} 3$ & 0.57 & $* *$ & 0.41 & ${ }^{* *}$ & 0.26 & * & 0.51 & $* *$ & 6.69 & $* *$ & 6.20 & $* *$ \\
\hline $\mathrm{P} 1 \times \mathrm{P} 4$ & -0.10 & $\mathrm{~ns}$ & 0.21 & ${ }^{* * *}$ & 1.10 & $* *$ & 0.17 & $*$ & 11.20 & ${ }^{* * *}$ & 1.96 & ${ }^{* *}$ \\
\hline $\mathrm{P} 1 \times \mathrm{P} 5$ & 0.58 & $* *$ & 0.69 & $* *$ & -0.48 & $* *$ & -0.03 & ns & 6.43 & $* *$ & -0.04 & ns \\
\hline $\mathrm{P} 1 \times \mathrm{P} 6$ & 0.27 & $* *$ & 0.22 & ${ }^{* *}$ & 0.53 & ${ }^{* * *}$ & 0.47 & ${ }^{* *}$ & -8.55 & ${ }^{* *}$ & -1.35 & $*$ \\
\hline $\mathrm{P} 1 \times \mathrm{P} 7$ & -0.97 & $* *$ & -0.81 & $* *$ & 0.07 & ns & 0.31 & $* *$ & -14.96 & $* *$ & -1.53 & * \\
\hline $\mathrm{P} 1 \times \mathrm{P} 8$ & 0.34 & $* *$ & 0.41 & ${ }^{* * *}$ & -0.46 & $* *$ & -0.03 & ns & -1.79 & ns & -2.85 & ${ }^{* *}$ \\
\hline $\mathrm{P} 2 \times \mathrm{P} 3$ & -0.46 & $* *$ & -0.70 & ${ }^{* *}$ & -0.05 & ns & 0.02 & ns & -2.69 & $*$ & 6.86 & $* *$ \\
\hline $\mathrm{P} 2 \times \mathrm{P} 4$ & 0.40 & $* *$ & 0.53 & $* *$ & 0.00 & ns & -0.05 & ns & -6.37 & $* *$ & 11.24 & $* *$ \\
\hline $\mathrm{P} 2 \times \mathrm{P} 5$ & -0.05 & $\mathrm{~ns}$ & -0.02 & ns & -0.58 & $* *$ & -0.20 & $* *$ & -10.12 & $* *$ & -0.45 & ns \\
\hline $\mathrm{P} 2 \times \mathrm{P} 6$ & -0.35 & $* *$ & -0.16 & ${ }^{*}$ & -0.11 & ns & -0.24 & ${ }^{* * *}$ & 13.10 & $* *$ & -4.95 & $* *$ \\
\hline $\mathrm{P} 2 \times \mathrm{P} 7$ & 0.39 & $* *$ & 0.58 & ${ }^{* *}$ & 0.73 & $* *$ & 1.11 & $* *$ & 10.18 & $* *$ & 1.91 & ${ }^{* *}$ \\
\hline $\mathrm{P} 2 \times \mathrm{P} 8$ & -0.30 & $* *$ & -0.53 & ${ }^{* * *}$ & 0.48 & *** & 0.35 & $* *$ & -6.49 & ${ }^{* *}$ & -1.78 & ${ }^{* *}$ \\
\hline $\mathrm{P} 3 \times \mathrm{P} 4$ & 0.99 & ${ }^{* * *}$ & 1.26 & ${ }^{* * *}$ & 0.03 & ns & 0.37 & ${ }^{* *}$ & 19.24 & ${ }^{* * *}$ & 9.73 & $* *$ \\
\hline $\mathrm{P} 3 \times \mathrm{P} 5$ & 0.60 & $* *$ & 0.40 & $* *$ & 0.52 & $* *$ & 1.08 & $* *$ & 8.45 & $* *$ & 17.62 & ${ }^{* *}$ \\
\hline $\mathrm{P} 3 \times \mathrm{P} 6$ & 0.35 & ${ }^{* * *}$ & 0.57 & ${ }^{* * *}$ & 0.06 & ns & 0.38 & ${ }^{* *}$ & 6.99 & ${ }^{* * *}$ & 9.52 & $* *$ \\
\hline $\mathrm{P} 3 \times \mathrm{P} 7$ & -0.22 & $* *$ & -0.21 & ${ }^{* * *}$ & -0.58 & ** & -1.39 & ${ }^{* *}$ & -10.28 & ${ }^{* *}$ & -8.41 & ${ }^{* *}$ \\
\hline $\mathrm{P} 3 \times \mathrm{P} 8$ & 0.16 & $*$ & 0.45 & $* *$ & -0.46 & $* *$ & -0.07 & ns & -3.26 & $* *$ & 6.95 & $* *$ \\
\hline $\mathrm{P} 4 \times \mathrm{P} 5$ & 0.49 & $* *$ & 0.20 & $*$ & -0.78 & $* *$ & -0.67 & $* *$ & -3.80 & $* *$ & -13.92 & $* *$ \\
\hline $\mathrm{P} 4 \times \mathrm{P} 6$ & -0.03 & $\mathrm{~ns}$ & -0.10 & ns & 0.07 & ns & 0.36 & $* *$ & 4.70 & $* *$ & 16.45 & ${ }^{* *}$ \\
\hline $\mathrm{P} 4 \times \mathrm{P} 7$ & -0.13 & ${ }^{*}$ & -0.01 & ns & 0.29 & ** & 0.35 & ${ }^{* *}$ & 14.84 & ${ }^{* *}$ & 0.17 & ns \\
\hline $\mathrm{P} 4 \times \mathrm{P} 8$ & 0.24 & $* *$ & 0.51 & ${ }^{* *}$ & -0.46 & ** & -0.80 & $* *$ & 14.44 & $* *$ & -4.08 & ${ }^{* *}$ \\
\hline $\mathrm{P} 5 \times \mathrm{P} 6$ & 0.11 & ns & -0.01 & ns & 0.02 & ns & 0.27 & ${ }^{* *}$ & 0.54 & ns & 6.93 & ${ }^{* *}$ \\
\hline $\mathrm{P} 5 \times \mathrm{P} 7$ & 0.14 & ${ }^{*}$ & 0.37 & ${ }^{* *}$ & 0.04 & ns & -0.14 & ${ }^{*}$ & 25.29 & ${ }^{* * *}$ & 15.87 & ${ }^{* *}$ \\
\hline $\mathrm{P} 5 \times \mathrm{P} 8$ & 0.66 & ${ }^{* *}$ & 0.55 & ${ }^{* *}$ & 0.32 & ** & 0.79 & ${ }^{* * *}$ & 18.20 & ${ }^{* *}$ & 28.76 & ${ }^{* *}$ \\
\hline $\mathrm{P} 6 \times \mathrm{P} 7$ & 1.23 & $* *$ & 1.07 & ${ }^{* *}$ & -0.50 & *** & -0.04 & ns & 12.72 & ${ }^{* * *}$ & 23.42 & $* *$ \\
\hline $\mathrm{P} 6 \times \mathrm{P} 8$ & -0.56 & $* *$ & -0.63 & ${ }^{* *}$ & -0.04 & ns & -0.23 & ** & -6.86 & ${ }^{* * *}$ & -11.38 & ** \\
\hline $\mathrm{P} 7 \times \mathrm{P} 8$ & 1.16 & + & 1.11 & ${ }^{* *}$ & 0.43 & 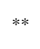 & 0.67 & 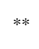 & 5.82 & $* *$ & 21.82 & $* *$ \\
\hline $\begin{array}{l}\text { S.E.(sij - } \\
\text { sji) }\end{array}$ & 0.06 & & 0.07 & & 0.09 & & 0.06 & & 0.51 & & 0.56 & \\
\hline
\end{tabular}

n.s.: non-significant, ${ }^{*} \mathrm{P}<0.05,{ }^{* *} \mathrm{P}<0.01$ 
Components of genetic variance

The components of genetic variance for studied traits are given in Table 7. The additive component (D) was not significant for all traits except plant height under salinity condition and number of grains per spike under normal condition which was positive and significant. On the other hand, the dominance components $\left(\mathrm{H}_{1}\right.$ and $\left.\mathrm{H}_{2}\right)$ were positive and significant for all studied traits. Furthermore, the value of $\mathrm{H}_{1}$ was higher than $\mathrm{D}$, proposing more contribution of the over-dominance effects in the inheritance of these traits compared to additive ones. This was confirmed by the net dominance component $\left(\mathrm{h}^{2}\right)$ which was positive and significant for all traits under both conditions except plant height and 100 grain weight under normal condition which was not significant. Average degree of dominance $\left(\mathrm{H}_{1} / \mathrm{D}\right)^{1 / 2}$ was higher than unity for all studied traits under both conditions which underline the presence of over-dominance gene effects for these traits. The dominance effects for these traits suggesting that selection in early generations may not be useful and it should be delayed to late generations. The importance of both additive and non-additive components in the inheritance of studied traits, with more presence for dominance components is in accordance with the results of Sharma et al. (2002), Ali et al. (2007), Eshghi \& Akhundova (2009), El-Seidy et al. (2010), Aghamiri et al. (2012), Tofiq et al. (2015), Patial et al. (2016) and Pesaraklu et al. (2016).

TABLE 7. Components of genetic variance for different traits under normal (Gh.) and salinity (Rs.) conditions.

\begin{tabular}{|c|c|c|c|c|c|c|c|c|}
\hline \multirow[b]{2}{*}{ Components } & \multicolumn{2}{|c|}{ Plant height (cm) } & \multicolumn{2}{|c|}{ No. of spikes/plant } & \multicolumn{2}{|c|}{ Spike length $(\mathbf{c m})$} & \multicolumn{2}{|c|}{ No. of grains/spike } \\
\hline & Gh. & Ras. & Gh. & Ras. & Gh. & Ras. & Gh. & Ras. \\
\hline D & 51.94 ns & $44.49^{* *}$ & $1.96 \mathrm{~ns}$ & $0.80^{\text {ns }}$ & 0.39 ns & $0.84^{\mathrm{ns}}$ & $36.84^{* *}$ & $51.17^{\mathrm{ns}}$ \\
\hline $\mathrm{H} 1$ & $263.90^{* *}$ & $251.94^{* *}$ & $33.93^{* *}$ & $43.91^{* *}$ & $2.91^{* *}$ & $4.03^{* *}$ & $208.53^{* *}$ & $370.27^{* *}$ \\
\hline $\mathrm{H} 2$ & $208.63^{* *}$ & $216.47^{* *}$ & $27.70^{* *}$ & $36.02^{* *}$ & $2.66^{* *}$ & $3.51^{* *}$ & $199.15^{* *}$ & $309.38^{* *}$ \\
\hline F & 101.51 ns & 40.58 ns & 5.27 ns & $0.10^{\mathrm{ns}}$ & 0.27 ns & 1.07 ns & 27.73 ns & $103.15^{\text {ns }}$ \\
\hline $\mathrm{h}^{2}$ & $0.01 \quad \mathrm{~ns}$ & $766.56^{* *}$ & $34.97^{* *}$ & $76.18^{* *}$ & $5.38^{* *}$ & $4.17^{* *}$ & $677.46^{* *}$ & $769.91^{* * *}$ \\
\hline E & 0.98 & 0.39 & 0.09 & 0.15 & 0.01 & 0.01 & 0.41 & 0.39 \\
\hline$(\mathrm{H} 1 / \mathrm{D})^{1 / 2}$ & 2.25 & 2.38 & 4.16 & 7.40 & 2.72 & 2.19 & 2.38 & 2.69 \\
\hline $\mathrm{H} 2 / 4 \mathrm{H} 1$ & 0.20 & 0.21 & 0.20 & 0.21 & 0.23 & 0.22 & 0.24 & 0.21 \\
\hline $\mathrm{KD} / \mathrm{KR}$ & 2.53 & 1.47 & 1.96 & 1.02 & 1.29 & 1.81 & 1.38 & 2.20 \\
\hline $\mathrm{h} 2 / \mathrm{H} 2$ & 0.00 & 3.54 & 1.26 & 2.12 & 2.02 & 1.19 & 3.40 & 2.49 \\
\hline $\mathrm{h}(\mathrm{n} . \mathrm{s})$ & 5.09 & 26.54 & 17.22 & 31.96 & 21.45 & 14.63 & 15.55 & 5.42 \\
\hline
\end{tabular}

n.s.: non-significant, ${ }^{*} \mathrm{P}<0.05,{ }^{* *} \mathrm{P}<0.01$

Egypt. J. Agron. Vol. 38, No. 3 (2016) 
TABLE 7. Cont.

\begin{tabular}{|c|c|c|c|c|c|c|}
\hline \multirow{2}{*}{ Components } & \multicolumn{2}{|c|}{ Grain weight/spike (g) } & \multicolumn{2}{|c|}{100 grain weight (g) } & \multicolumn{2}{|c|}{ Grain yield/plant (g) } \\
\hline & Gh. & Ras. & Gh. & Ras. & Gh. & Ras. \\
\hline D & 0.19 ns & $0.31 \quad$ ns & $0.18 \quad \mathrm{~ns}$ & 0.07 ns & 26.17 ns & 6.05 \\
\hline H1 & $1.72^{* *}$ & $2.04^{* *}$ & $0.89^{* *}$ & $1.32^{* *}$ & $728.17^{* *}$ & 827.35 \\
\hline H2 & $1.48^{* *}$ & $1.65^{* *}$ & $0.82^{* *}$ & $1.24^{* *}$ & $603.46^{* *}$ & 701.13 \\
\hline $\mathbf{F}$ & 0.27 ns & 0.59 ns & $0.10^{\mathrm{ns}}$ & $0.11^{\text {ns }}$ & $45.44^{\mathrm{ns}}$ & 25.25 \\
\hline $\mathbf{h}^{2}$ & $2.72^{* *}$ & $3.02^{* *}$ & $0.00^{\mathrm{ns}}$ & $0.67^{* *}$ & $990.55^{* *}$ & 1517.22 \\
\hline $\mathbf{E}$ & 0.00 & 0.00 & 0.01 & 0.00 & 0.34 & 0.33 \\
\hline$(\text { H1/D })^{1 / 2}$ & 3.00 & 2.56 & 2.22 & 4.41 & 5.28 & 11.70 \\
\hline H2/4H1 & 0.22 & 0.20 & 0.23 & 0.24 & 0.21 & 0.21 \\
\hline KD/KR & 1.62 & 2.19 & 1.29 & 1.44 & 1.39 & 1.43 \\
\hline h2/H2 & 1.83 & 1.83 & 0.00 & 0.54 & 1.64 & 2.16 \\
\hline $\mathbf{h}(\mathbf{n} . \mathbf{s})$ & $\begin{array}{r}16.7 \\
6 \\
\end{array}$ & 11.64 & 27.17 & 4.62 & 25.85 & 23.35 \\
\hline
\end{tabular}

n.s.: non-significant, ${ }^{*} \mathrm{P}<0.05,{ }^{* *} \mathrm{P}<0.01$

The relative frequency of dominant to recessive alleles in the genotypes (F) was positive and insignificant for all studied traits, revealing to abundance of dominant alleles than recessive ones in the parents, as well as the important role of dominant genes for the studied traits (Table 7). This is corresponding with the proportion of dominance and recessive genes $(\mathrm{KD} / \mathrm{KR})$ in the parents which was more than the unity, implying that the dominant genes were more frequent than recessive ones for all studied traits under both conditions. Also this result is supported by the estimates of the ratio $\mathrm{H}_{2} / 4 \mathrm{H}_{1}$ which were less than 0.25 for all traits under both conditions, confirming the unequal distribution of positive and negative alleles among the parents. The environmental component (E) was high for plant height, number of spikes per plant, number of grains per spike and grain yield per plant under both conditions revealing the importance of environmental factors in expression of these traits, while low value was assigned for other traits. These results are in agreement with the finding of Rohman et al. (2006), Eshghi \& Akhundova (2009), Aghamiri et al. (2012), Metwali et al. (2014) and Pesaraklu et al. (2016).

Phenotypic selection efficiency depends on the proportion of narrow sense heritability, where it is directly relative to additive genetic variance (Falconer et al., 1996). Narrow sense heritability values were detected for all studied traits and changed from environment to another, it ranged from low to moderate (4.62\% to 31.96$)$. Especially under salinity condition the values were lower than normal one for grain yield and its components (Table 7). The low values due to excess of dominance effect as well as great effect of the environmental factors in the genetic control of these traits. Similar low narrow sense heritability was reported by Rohman et al. (2006) for number of spikes per plant, Eshghi \& Akhundova (2009) for number of grains per spike, Pesaraklu et al. (2016) for plant height, spike length, grain weight per 
spike and 100 grain weight and Tofiq et al. (2015) and Ahmadi et al. (2016) for grain yield per plant.

\section{Wr-Vr Graph}

The regression graphs of covariance (Wr) and variance (Vr) were constructed for further understanding the genetic nature of parental variation. The graphs for studied traits under normal condition (Ghazala) are shown in Fig. 1, and under salinity condition (Ras-Sudr) are shown in Fig. 2. Under normal condition, the regression line passed below the origin in all studied traits except plant height and 100 grain weight, the line passed through and above the origin, respectively. This indicated to presence of complete dominance genes effects in the inheritance of plant height, partial dominance for 100 grain weight and over dominance for the remaining traits. While under salinity condition, the regression line passed below the origin in plant height, grain weight per spike and 100 grain weight revealing to presence of over dominance in the inheritance of these traits. In number of spikes per plant, spike length and grain yield, the line passed over the origin revealing the presence of partial dominance. While in number of grains per spike, the line passed through the origin revealing the presence of complete dominance.

Under normal condition the distribution of parents along the regression line revealed that $\mathrm{P} 4$ possess maximum number of dominant alleles for plant height (Fig. 1). Also P1 and P2 carry dominant alleles for number of spikes per plant, grain weight per spike and grain yield per plant, P1 and P3 for spike length, P2 and P5 for number of grains per spike, and P3 and P6 for 100 grain weight. Otherwise P3 carries maximum number of recessive alleles for plant height, P4 and P5 carry recessive alleles for number of spikes per plant, P5 for spike length, P6 for number of grains per spike, P8 for grain weight per spike, P1 and P4 for 100 grain weight and P4, P5 and P7 for grain yield per plant.

While under salinity condition P2 possesses maximum number of dominant alleles for plant height and number of grains per spike (Fig. 2). Also P1 and P4 carry dominant alleles for number of spikes per plant, P1 and P3 for spike length, P1 for grain weight per spike and 100 grain weight, and $\mathrm{P} 1$ and P2 for grain yield per plant. While P3 carries maximum number of recessive alleles for plant height, as well as P8 carry recessive alleles for number of spikes per plant and grain weight per spike, P7 for spike length, number of grains per spike and 100 grain weight, and P5 and P8 for grain yield per plant. These results reveal to genetic diversity of parents, therefore, it could be expected that the combination of these genotypes lead to high degree of heterosis and production high values of the traits. Similar finding was reported by Jana (1975), Abdel-Sabour et al. (1990), Madić et al. (2005), Rohman et al. (2006), Eshghi \& Akhundova (2009), Aghamiri et al. (2012) and Ciulca et al. (2012). 


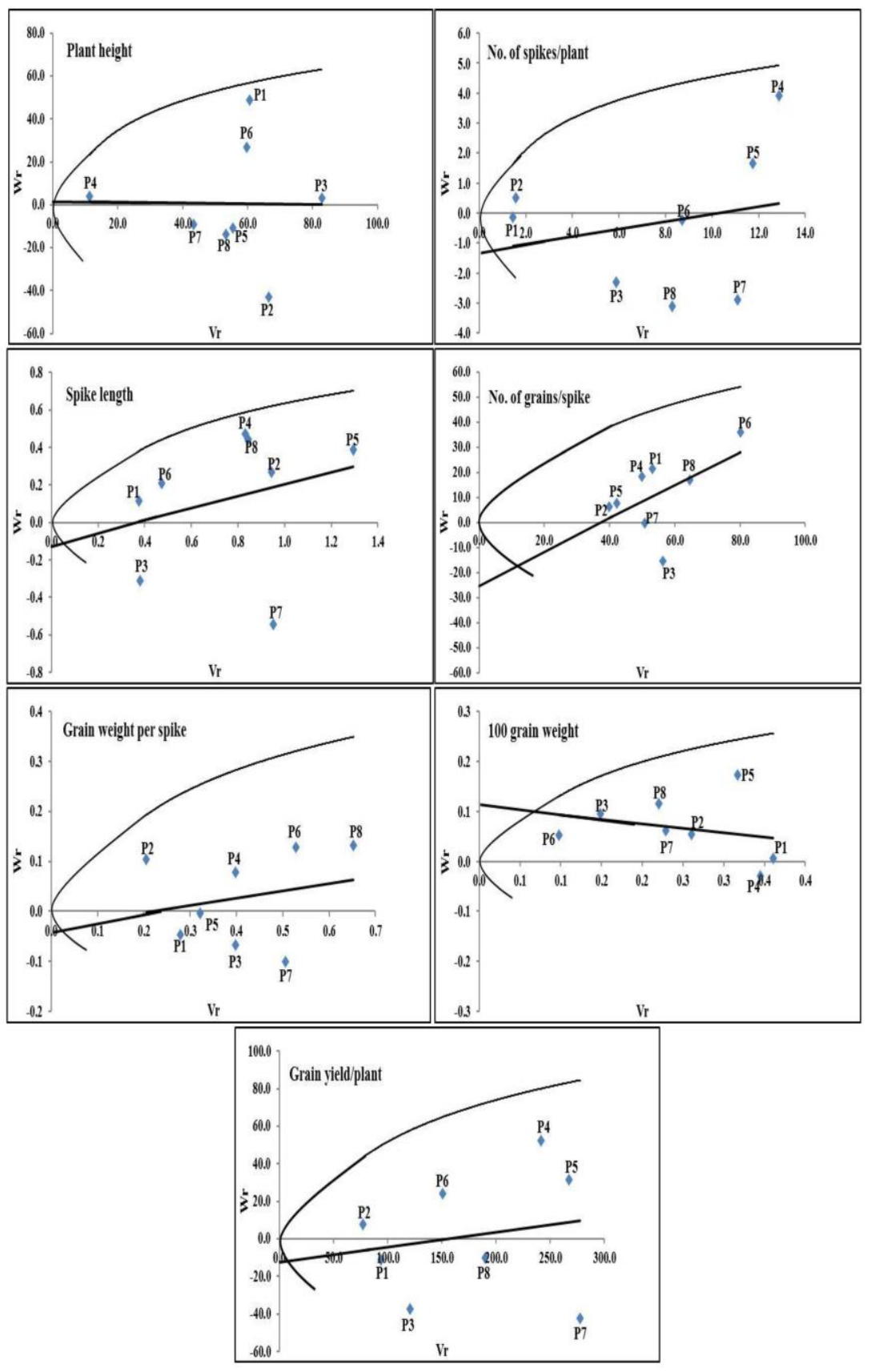

Fig. 1. Wr-Vr graph for different agronomic traits of parental barley genotypes under normal condition. 


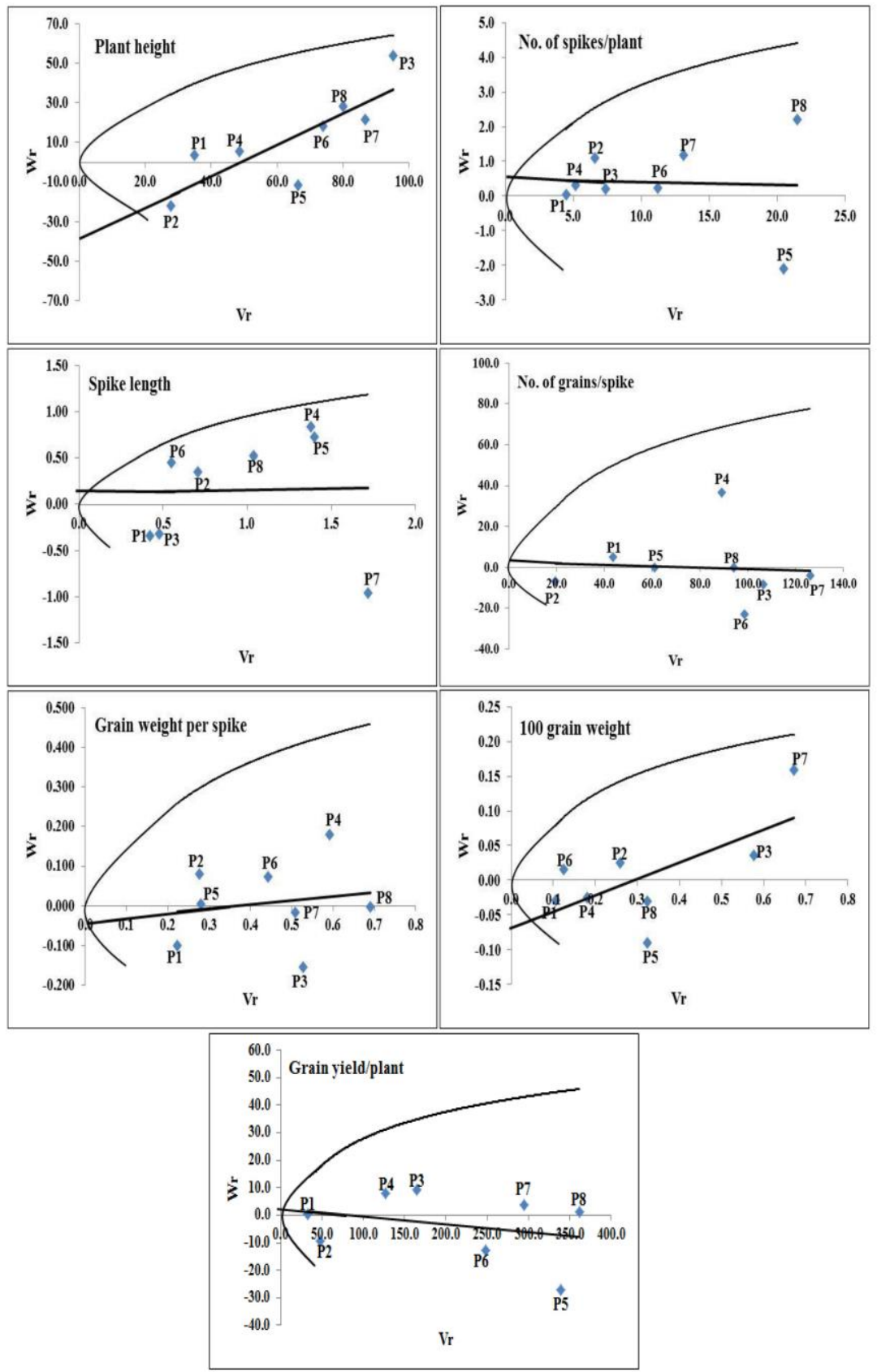

Fig. 2. Wr-Vr graph for agronomic different traits of parental barley genotypes under salinity condition.

Egypt. J. Agron. Vol. 38, No. 3 (2016) 


\section{Conclusion}

This work focused on eight six-rowed hulled spring barley genotypes and their crosses which could be exploited further in barley breeding to improve production under normal and salinity conditions. Most of used genotypes exhibited good performance and combining ability for different traits. Especially P3 and P6 under salinity condition, P4 and P8 under normal one and P2, P5 and P7 under both conditions. At least one of these genotypes participated in the cross combinations which presented high performance and SCA values for studied traits. The best specific crosses were, $\mathrm{P} 3 \times \mathrm{P} 5, \mathrm{P} 3 \times \mathrm{P} 8$ and $\mathrm{P} 5 \times \mathrm{P} 8$ under salinity, and $\mathrm{P} 2 \times \mathrm{P} 7, \mathrm{P} 3 \times \mathrm{P} 4, \mathrm{P} 4 \times \mathrm{P} 7, \mathrm{P} 5 \times \mathrm{P} 7 \mathrm{P} 6 \times \mathrm{P} 7$ and $\mathrm{P} 7 \times \mathrm{P} 8$ under both conditions. Thus; these parental genotypes and their crosses could be further used in developing segregating populations in barley breeding programs, to improve grain yield and contributing traits under both conditions.

The obtained results by using Griffing and Hayman approach illustrated the importance of both additive and non-additive genetic components in the inheritance of studied traits, with more presence for dominance components. Also $\mathrm{Wr}-\mathrm{Vr}$ graphs supported this result, where, the dominance effect was excess for all studied traits. On the other hand, the distribution of dominant and recessive alleles in the parental material for all traits reveals to genetic diversity of parents.

From all forgoing results, it could be concluded that the above mentioned cross combinations lead to high degree of heterosis and higher production for improving yield and its components when exploited it in future barley breeding programs. Otherwise, the presence of dominance effect as well as great effect of the environmental factors in the genetic control of studied traits reflected moderately low narrow sense heritability estimates. Which reveals that selection should be delayed to later generation.

Acknowledgements: The authors thank Dr. Hassan Oda Awad for his valuable reviewing the manuscript.

\section{Reference}

Abdel-Sabour, M.S., Alkaddoussi, A.R. and Hassan, E.E. (1990) Genetic behaviour of some yield components in barley. Zagazig. J. Agric. Res. 17, 23-30.

Aghamiri, S., Mostafavi, K. and Mohammadi, A. (2012) Genetic study of agronomic traits in barley based diallel cross analysis. Advances in Environmental Biology, 6, 62-68.

Ahmadi, J., Vaezi, B. and Pour-Aboughadareh, A. (2016) Analysis of variability, heritability, and interrelationships among grain yield and related characters in barley advanced lines. Genetika. 48, 73-85. 
Ahmed, I.A., El-Sherbini, A.M., El-Bawab, A.M., El-Moselhy, M.A. and Salem, E.M. (1998) Diallel analysis for yield and yield component in barley. Agric .Sci. Mansoura Univ. 22, 2971-2979.

Ali, A., Mageed, M., Ahmed, I. and Mariey, S. (2007) Genetic and molecular studies on barley salt tolerance. In: African Crop Science Conference Proceedings, Vol. 8, pp. 669-682.

Biel, W., and Jacyno, E. (2013) Chemical composition and nutritive value of spring hulled barley varieties. Bulgarian J. Agr. Sci. 19, 721-727

Ciulca, S., Adriana, C., Madosa, E. and Giancarla, V. (2012) Diallel analysis of variance-covariance regression for spike length in six-row winter barley. J. Hortic. For. Biotechnol. 16, 82-86.

El - Hendawy, S., Ruan, Y., Hu, Y. and Schmidhalter, U. (2009) A comparison of screening criteria for salt tolerance in wheat under field and controlled environmental conditions. J. Agron. Crop Sci. 195, 356-367.

El-Seidy, E.H., El-Moselhey, M.A., El-Gammaal, A.A. and El-Naggar, A.A. (2010) Combining ability and genetic component analyses for barley genotypes (Horduem vulgare L.) under stress and non-stress environments. J. Plant Prod. Mansoura Univ. 1, 1451-1466.

Eshghi, R. and Akhundova, E. (2009) Genetic analysis of grain yield and some agronomic traits in hulless barley. Afr. J. Agric. Res. 4, 1464-1474.

Falconer, D.S., Mackay, T.F. and Frankham, R. (1996) "Introduction to Quantitative Genetics" ( $4^{\text {th }}$ ed). Trends in Genetics, 12, 280.

Faostat, D. (2016) Food and Agriculture Organization of the United Nations. Statistical database.

Griffing, B. (1956) Concept of general and specific combining ability in relation to diallel crossing systems. Aust. J. Biol. Sci. 9, 463-493.

Hallauer, A. and Miranda, J. (1988) "Quantitative Genetics in Maize Breeding" Iowa State Univ. Press. Ames, IA.

Hayman, B. (1954a) The analysis of variance of diallel tables. Biometrics, 10, 235-244.

Hayman, B. (1954b) The theory and analysis of diallel crosses. Genetics, 39, 789.

Jana, S. (1975) Genetic analysis by means of diallel graph. Heredity, 35, 1-19.

Jinks, J. and Hayman, B. (1953) The analysis of diallel crosses. Maize Genetics Cooperation Newsletter, 27, 48-54.

Kulshreshtha, N. and Singh, K. (2011) Combining ability studies in wheat (Triticum aestivum L.) for genetic improvement under salt stress. J. Wheat Res. 3(2),22-26 
Madić, M., Paunović, A., Djurović, D., Kraljević-Balalić, M. and Knežević, D. (2005) The analysis of gene effect in the inheritance of kernel number per spike in barley hybrid. Genetika. 37, 261-269.

Madić, M., Djurović, D., Knezevic, D., Paunović, A. and Tanasković, S. (2014) Combining abilities for spike traits in a diallel cross of barley. J. Cent. Eur. Agric. 15, 108-116.

Mather, K. and Jinks, J.L. (1982) Diallels. In "Biometrical Genetics", pp. 255-291. Springer.

Metwali, E.M.R., Abd El-Haleem, S.H.M., EL-Saeid, R.A.R. and Kadasa, N.M.S. (2014) An investigation of gene action on different traits of barley (Hordeum vulgare L.) using partial diallel crosses system. Life Sci. J. 11, 64-71.

Patial, M., Pal, D. and Kumar, J. (2016) Combining ability and gene action studies for grain yield and its component traits in barley (Hordeum vulgare L.). SABRAO J. Breed. Genet. 48, 90-96.

Pesaraklu, S., Soltanloo, H. Ramezanpour, S., KalateArabi, M. and Nasrollah NejadGhomi, A. (2016) An estimation of the combining ability of barley genotypes and heterosis for some quantitative traits. Iran Agric. Res. 35, 73-80.

Poehlman, J.M. (1985) Adaptation and distribution. In: "Barley Agronomy", Rasmusson, D. C. (Ed.), pp. 1-17. No 26, Madison, WI, USA, Amer. Soc. Agron.

Potla, K., Bornare, S., Prasad, L., Prasad, R. and Madakemohekar, A. (2013) Study of heterosis and combining ability for yield and yield contributing traits in barley (Hordeum vulgare L.). The Bioscan, 8, 1231-1235.

Qu, Z., Li, L., Luo, J., Wang, P., Yu, S., Mou, T., Zheng, X. and Hu, Z. (2012) QTL mapping of combining ability and heterosis of agronomic traits in rice backcross recombinant inbred lines and hybrid crosses. PLoS One, 7, e28463.

Rohman, M., Sultana, R., Podder, R., Islam, A.T., Islam, M.K. and Islam, M. (2006) Nature of gene action in barley (Hordeum vulgare L.). Asian J. Plant Sci. 5, 170173.

Saad, F., El-Mohsen, A.A., El-Shafi, M.A. and Al-Soudan, I. (2013) Genetic behavior of grain yield and its components in barley crosses under water stress and non-stress conditions. Scientia, 1, 45-55.

Salgotra, R., Gupta, B. and Singh, P. (2009) Combining ability studies for yield and yield components in basmati rice. Oryza Int. J. Rice. 46, 12-16.

Sharma, Y., Sharma, S., Joshi, P. and Sain, R. (2002) Combining ability analysis for yield and yield contributing characters in six-rowed barley. SABRAO J. Breed. Genet. 34, 55-64.

Syukur, M., Sujiprihati, S. and Yunianti, R. (2010) Analysis using Hayman method to study genetic parameters of yield components in pepper (Capsicum annuum L.). Hayati J. Biosci. 17, 183-188. 
Tofiq, S.E., Amin, T.N.H, Abdulla, S.M.S. and Abdulkhaleq, D.A. (2015) Genetic analysis in some barley varieties and their hybrids in F2 generation. Int. J. Plant Ani. Environ. Sci. 5, 208-217.

Walejkl, R. and Rusell, W. (1977) Application of combining ability in plant breeding. Crop Sci. 17, 747-751.

Yilmaz, R. and Konak, C. (2000) Heterotic effects regarding salt tolerance in some characters of barley (Hordeum vulgare L.). Turk. J. Agric. For. 24, 643-648.

Zecevic, V., Boskovic, J., Dimitrijevic, M. and Petrovic, S. (2010) Genetic and phenotypic variability of yield components in wheat (Triticum aestivum L.). Bul. J. Agric. Sci. 16, 422-428.

Zhang, X., Lv, L., Lv, C., Guo, B. and Xu, R. (2015) Combining ability of different agronomic traits and yield components in hybrid barley. http://dx. doi. Org/10.1371/ journal. pone. 0126828

(Received $3 / 10$ /2016;

accepted $22 / 12 / 2016$ ) 


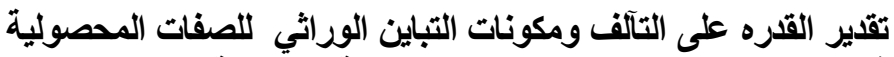 فى الشعير الربيعى تحت الظروف الطبيعية والمبلية الثية
}

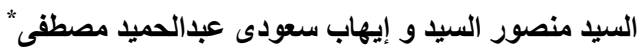

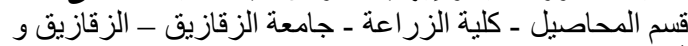

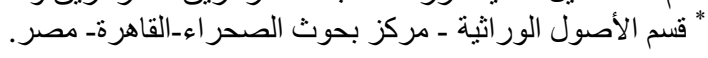

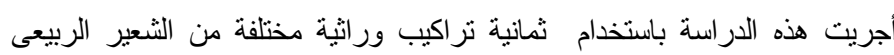

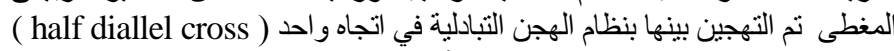

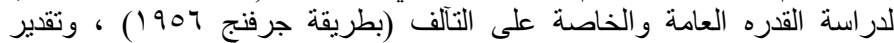

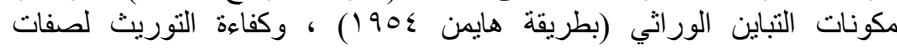

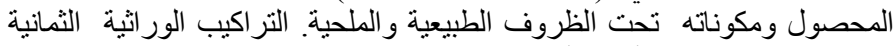

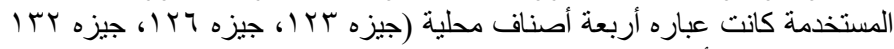

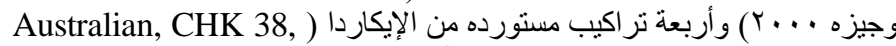
CHK 2 and CHK 53

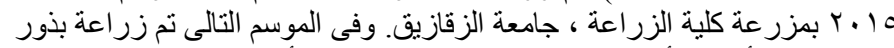

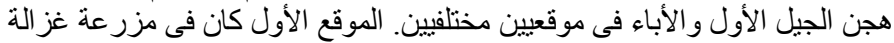

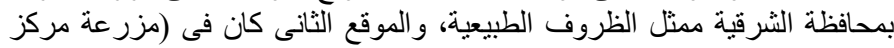

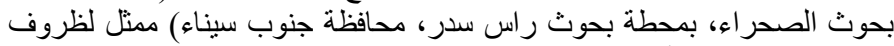

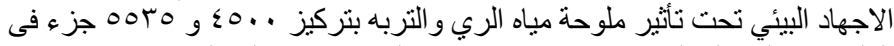

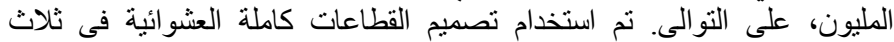

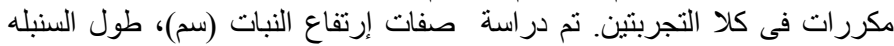

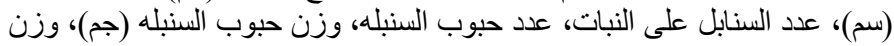
المائة حبه (جم) و محصول حلى لبوب النبات الفردي (جم).

إثار تحليل التباين إلى وجود فروق عالية المعنوية بين الأباء وكذلك بين هجن

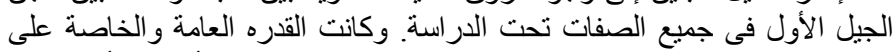

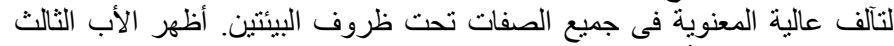
(Australian)

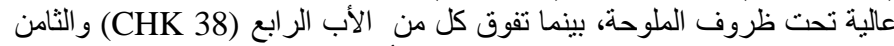

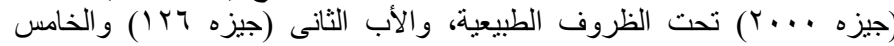

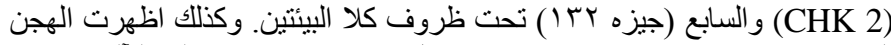

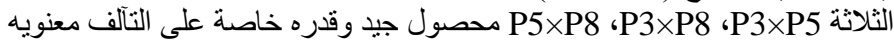
وموجبه لصفة المحصول و الصفات المساهمه تحت ظروف الملوحة، وخمسة هجن

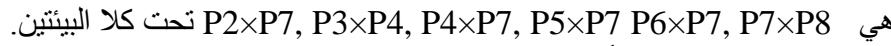

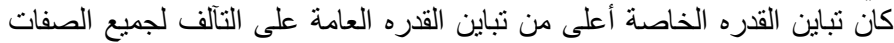

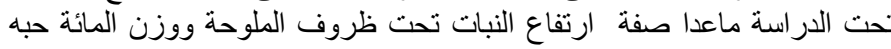
تحت الظروف الطبيعية.

أظهرت نتائج مكونات التباين الوراثى (Hayman 1954) مساهمة كبيره

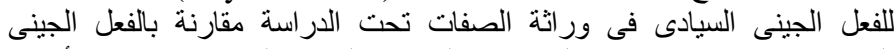

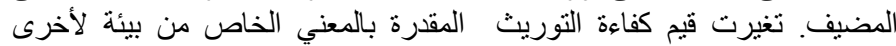

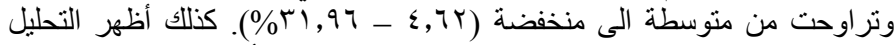

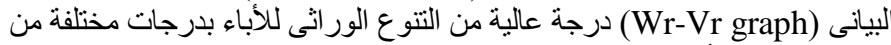
السيادة وتوزيع الأليلات السائدة و المتنحية لكل الصفات الصنات تحت الدر اسة فى كلا البيئتين. 